\title{
Spububaúde
}

\section{A pandemia de COVID-19 e os impactos na saúde pública do estado do Rio de Janeiro}

\author{
The pandemic of COVID-19 and the impacts on public health in the state of Rio de Janeiro
}

\author{
Tatiana Souza da Silva Werle ${ }^{1^{*} \bullet}$, Antonio Rodrigues de Andrade ${ }^{2}$
}

\begin{abstract}
${ }^{1}$ Mestranda do Programa de Pós-Graduação em Saúde e Tecnologia no Espaço Hospitalar da Universidade Federal do Estado do Rio de Janeiro, Rio de Janeiro, Rio de Janeiro, Brasil. ${ }^{2}$ Professor Titular do Programa de Pós-Graduação em Saúde e Tecnologia no Espaço Hospitalar da Universidade Federal do Estado do Rio de Janeiro, Rio de Janeiro, Rio de Janeiro, Brasil. *Autor para correspondência. Email: tatissil@uol.com.br
\end{abstract}

\begin{abstract}
Resumo: Introdução: O novo coronavírus SARS-CoV-2, causador da doença COVID-19, apresenta um espectro clínico variável, podendo causar infecções assintomáticas e oligossintomáticas, mas que podem evoluir necessitando de atendimento hospitalar e até mesmo de suporte ventilatório. Desta forma, o estudo tem por objetivo descrever por meio de indicadores o impacto da pandemia sobre a disponibilidade de leitos de internação nos municípios do Estado do Rio de Janeiro. Material e métodos: Foi realizado um estudo ecológico espacial transversal, com abordagem quantitativa. Os dados foram baseados nos boletins epidemiológicos para acompanhamento dos casos de COVID-19 nos municípios do estado do Rio de Janeiro, relativos ao período de 01 de março a 13 de junho de 2020, onde foram analisadas 9 regiões de saúde que englobam os 92 municípios do estado. Resultados e discussão: Observou-se que a região de maior incidência, equivalente a 3,47/100.000 habitantes, corresponde a região da Baixada Litorânea, contudo considerando a população absoluta o maior número de casos ocorreu na região Metropolitana I, com 197.445, sendo 144.199 no município do Rio de Janeiro. Além da análise descritiva foi calculada a correlação de Pearson e o resultado foi de 0,98 , ao nível de 5\%, o qual confirma que o valor da correlação é significativo. Considerações finais: Fica evidenciado que o Estado deve intensificar as políticas educativas que busquem conscientizar a população sobre a forma de disseminação do vírus e ampliar as estratégias para identificação rápida do vírus, isolamento e tratamento precoce, em virtude de não haver disponibilidade de leitos de internação para atender todos os casos de COVID que evoluam com complicações.
\end{abstract}

Palavras-chave: COVID-19, leitos, políticas públicas, saúde.

\begin{abstract}
Introduction: The new SARS-CoV-2 coronavirus, which causes COVID-19 disease, has a variable clinical spectrum, which can cause asymptomatic and oligosymptomatic infections, but which may evolve requiring hospital care and even ventilatory support. Thus, the study aims to describe by means of indicators the impact of the pandemic on the availability of hospital beds in the municipalities of the State of Rio de Janeiro. Material and methods: A cross-sectional spatial ecological study was carried out, with a quantitative approach. The data were based on epidemiological bulletins for monitoring cases of COVID19 in the municipalities of the State of Rio de Janeiro, for the period from March 1 to June 13, 2020, where 9 health regions covering 92 municipalities were analyzed of State. Results and discussion: It was observed that the region with the highest incidence, equivalent to $3,47 / 100.000$ population, corresponds to the Baixada Litorânea region, however considering the absolute population the largest number of cases occurred in Metropolitan I, with 197,445, being 144,199 in the municipality of Rio de Janeiro. In addition to the descriptive analysis, Pearson's correlation was calculated and the result was 0.98 , at the $5 \%$ level, which confirms that the correlation value is significant. Final considerations: It is evident that the State should intensify educational policies that seek to raise the population's awareness of how to spread the virus and expand strategies for rapid virus identification, isolation and early treatment, due to the lack of availability hospitalization beds to attend all cases of COVID that evolve with complications.
\end{abstract}

Keywords: COVID-19, beds, public policies, health.

\section{Introdução}

O novo coronavírus, denominado SARS-CoV-2, causador da doença COVID-19, foi detectado em 31 de dezembro de 2019 em Wuhan, na China. Em 9 de janeiro de 2020, a Organização Mundial da Saúde (OMS) confirmou a circulação do novo coronavírus. No dia seguinte, a primeira sequência do SARS-CoV-2 foi publicada por pesquisadores chineses. Em 16 de janeiro, foi notificada a primeira importação em território 
japonês. No dia 21 de janeiro, os Estados Unidos reportaram seu primeiro caso importado. Em 30 de janeiro, a OMS declarou a epidemia uma emergência internacional. Ao final do mês de janeiro, diversos países já haviam confirmado importações de caso, incluindo Estados Unidos, Canadá e Austrália. No Brasil, em 7 de fevereiro, havia 9 casos em investigação, mas sem registros de casos confirmados (Brasil, 2020a).

A COVID-19 apresenta um espectro clínico variando de infecções assintomáticas a quadros graves. De acordo com a OMS, a maioria (cerca de 80\%) dos pacientes com COVID-19 podem ser assintomáticos ou oligossintomáticos (poucos sintomas), e aproximadamente $20 \%$ dos casos detectados requer atendimento hospitalar por apresentarem dificuldade respiratória, dos quais aproximadamente $5 \%$ podem necessitar de suporte ventilatório (Brasil, 2020b).

Os sinais/sintomas iniciais da doença lembram um quadro gripal comum, mas variam de pessoa para pessoa, podendo se manifestar de forma branda, em forma de pneumonia, pneumonia grave e SRAG. A maior parte das pessoas infectadas apresenta a forma leve da doença, com alguns sintomas como mal-estar, febre, fadiga, tosse, dispneia leve, anorexia, dor de garganta, dor no corpo, dor de cabeça ou congestão nasal, sendo que algumas também podem apresentar diarreia, náusea e vômito. Idosos e imunossuprimidos podem ter uma apresentação atípica e agravamento rápido, o que pode causar a morte, principalmente dos idosos e indivíduos com comorbidades preexistentes (Iser et al., 2020).

O primeiro caso do novo Coronavírus (Covid-19) no estado do Rio de Janeiro, ocorreu em 05 março de 2020. Tratava-se de jovem, de 27 anos, moradora de Barra Mansa, no Sul Fluminense. A vítima havia viajado no dia 9 de fevereiro para a Europa, de onde retornou dia 23. Ela esteve na Itália (em Milão e na Lombardia) e Alemanha (Brasil, 2020c).

Em 12 de março de 2020, foi identificado o primeiro caso de transmissão local no estado, um homem de 73 anos. No dia 19 de março do mesmo ano foi identificado o primeiro óbito, tratava-se de uma mulher de 68 anos que trabalhava como empregada doméstica e que teve contato com a empregadora que havia retornado da Itália e testado positivo para o COVID-19 (Brasil, 2020b; Iser et al., 2020).

Destaca-se o fato de o prognóstico de cura dos casos mais graves está relacionado à disponibilidade de recursos de saúde, tais como leitos de unidade de terapia intensiva (UTI), respiradores mecânicos e quantitativo da força de trabalho em saúde (Emanuel et al., 2020).

Este cenário complexo impõe desafios adicionais à vigilância epidemiológica, às relações internacionais e à programação de políticas públicas, sobretudo por meio de medidas que reduzam as desigualdades de acesso aos sistemas de saúde e a condições estruturais para o autocuidado (Rafael et al., 2020).

A consolidação dos dados sobre casos e óbitos por COVID-19, coletados e disponibilizados pelas Secretarias Estaduais de Saúde, vem sendo realizada desde o início da pandemia pelo Ministério da Saúde brasileiro. Isso permite o conhecimento da dinâmica da doença e, consequentemente, o estabelecimento de políticas para desacelerar o incremento no número de casos.

Diante da situação retratada emergiu a questão fundante da pesquisa: O quantitativo de leitos de internação é suficiente para atender a demanda de COVID no estado do Rio de Janeiro?

O objetivo da pesquisa foi descrever por meio de indicadores o impacto da pandemia sobre a disponibilidade de leitos de internação nos municípios do estado do Rio de Janeiro.

\section{Material e métodos}

Trata-se de um estudo ecológico espacial transversal com abordagem quantitativa cuja as unidades de análise foram as Regiões de Saúde do Estado do Rio de Janeiro. Segundo o Ministério da Saúde, conceitua-se Região de Saúde como o espaço geográfico continuo, formado pela união de municípios limítrofes, segundo identidades culturais, econômicas sociais. Além disso, consideram-se as redes de comunicação e infraestrutura de transportes compartilhados, com o objetivo de integrar a organização, o planejamento e a execução de ações e serviços de saúde (Brasil, 2020d).

Os métodos de análise espacial vêm sendo utilizados principalmente em estudos ecológicos, na detecção de aglomerados espaço-temporais voltados para o planejamento e avaliação do uso de serviços de saúde. Em situações de surtos e epidemias, a análise espacial é uma importante ferramenta para identificação de áreas de maior risco, servindo como suporte para implementação de medidas de controle (Cavalcante \& Abreu, 2020).

A abordagem quantitativa é caracterizada pelo emprego da quantificação, tanto nas modalidades de coleta de informações quanto no tratamento delas por meio de técnicas estatísticas. Busca a validação das hipóteses mediante a utilização de dados estruturados, estatísticos, com análise de um grande número de casos representativos, recomendando um curso final da ação (Richardson, 1999; Mattar, 2001). 
Os dados referem-se ao período de 01 março a 13 junho de 2020. Foram consultadas as bases do Departamento de Informática do SUS - DataSUS, Secretaria de Saúde do Governo do Estado do Rio de Janeiro - SES-RJ e Ministério da Saúde - MS. As variáveis analisadas foram: população total, número de casos confirmados, número de óbitos, número de leitos de internação.

Foram avaliados os 92 municípios do estado do Rio de Janeiro considerando as 9 regiões de saúde, definidas pelo Plano Diretor de Regionalização de Saúde do Rio de Janeiro, distribuídas da presente forma: Baia da Ilha Grande, Baixada Litorânea Centro-Sul, Médio Paraíba, Metropolitana I, Metropolitana II, Noroeste, Norte e Serrana (Mattar, 2001).

A amostra final contou com as informações de 92 municípios, acerca da população estimada, leitos de internação, casos COVID notificados e óbitos. Além da análise descritiva foi calculada a correlação de Pearson, o resultado foi de 0,98 , ao nível de $5 \%$, afirmando que o valor da correlação é significativo.

Todos os gráficos e testes foram realizados no programa estatístico R (R version 3.0.2@ 2013). O nível de significância estatístico considerado foi de 0,05.

\section{Resultados e discussão}

No período de 01 de março a 13 de junho de 2020, foram registrados casos e óbitos confirmados por COVID19 no estado do Rio de Janeiro (Brasil, 2020b).

Diante da magnitude da doença foram analisados os dados quanto ao número de leitos disponíveis e o número de vítimas afetadas pelo vírus SARS-CoV-2.

Destaca-se que os fatores que determinam os padrões de transmissão, ocorrência e mortes associados à doença são amplos e diversos, e seus impactos, atuais e futuros, afetam de modo abrangente a economia e a sociedade. Os efeitos da pandemia são heterogêneos e apresentam forte dimensão territorial, dado o importante papel dos governos subnacionais em relação às medidas de contenção, atenção à saúde, serviços sociais, e às despesas públicas, colocando-os na linha de frente da gestão da crise. Além disso, existem muitas incertezas quanto à dinâmica da pandemia em um cenário de profundas desigualdades, elevada carga de doença e precárias condições de vida das populações (Brasil, 2009). As tabelas 1 e 2 demonstram o perfil de casos por COVID-19 nos municípios do estado do Rio de Janeiro, considerando as regiões de saúde:

Tabela 1. Perfil de casos por COVID-19 nas Regiões de Saúde, período (01/03 a 13/06/20).

\begin{tabular}{ccccccc}
\hline Regiões de saúde & $\begin{array}{c}\text { População } \\
\text { absoluta }\end{array}$ & $\begin{array}{c}\text { Casos } \\
\text { confirmados }\end{array}$ & Óbitos & $\begin{array}{c}\text { Leitos clínicos } \\
\text { (SUS) }\end{array}$ & Incidência & Letalidade \\
\hline Baia da Ilha Grande & 295.944 & 9.695 & 308 & 259 & 0.03276 & 0.03176 \\
Baixada Litorânea & 855.444 & 14.861 & 604 & 419 & 0.03479 & 0.02065 \\
Médio Paraíba & 918.097 & 23.536 & 774 & 1.014 & 0.02966 & 0.02444 \\
Centro Sul & 342.078 & 10.146 & 204 & 290 & 0.02789 & 0.02960 \\
Metropolitana I & 10.542 .254 & 197.945 & 17.172 & 3.909 & 0.01737 & 0.04333 \\
Metropolitana II & 2.131 .058 & 49.816 & 2.034 & 1.134 & 0.02563 & 0.03289 \\
Noroeste & 349.417 & 12.155 & 251 & 238 & 0.02610 & 0.03452 \\
Norte & 955.122 & 26.619 & 788 & 631 & 0.02338 & 0.04083 \\
Serrana & 976.775 & 25.494 & 880 & 733 & 0.01878 & 0.08675 \\
\hline
\end{tabular}

Tabela 2. Perfil de casos por COVID-19 nas Regiões de Saúde, período (01/03 a 13/06/20).

\begin{tabular}{|c|c|c|c|c|c|}
\hline Regiões de saúde & Min. & Mediana & Média & Máx. & Desvio Padrão \\
\hline Baia da Ilha Grande & 1061 & 1441 & 3232 & 7193 & 3435.873 \\
\hline Baixada Litorânea & 281 & 1394 & 1651 & 3667 & 1047.846 \\
\hline Médio Paraíba & 30 & 832 & 1961 & 9255 & 2697.571 \\
\hline Centro Sul & 176.0 & 429.0 & 922.4 & 3942.0 & 1074.52 \\
\hline Metropolitana I & 649 & 3782 & 16495 & 144199 & 40408.14 \\
\hline Metropolitana II & 455 & 4670 & 7117 & 19139 & 7619.2 \\
\hline Noroeste & 171.0 & 634.5 & 868.2 & 3763.0 & 910.0228 \\
\hline Norte & 313.0 & 870.5 & 3327.4 & 11355 & 4603.065 \\
\hline Serrana & 85.0 & 479.0 & 1593.4 & & 10118 \\
\hline
\end{tabular}

Fonte: DataSUS, SES-RJ e MS, 2020.

Conforme podemos observar a região de maior incidência com 3,47/100.000 habitantes, corresponde a região da Baixada Litorânea, contudo considerando a população absoluta o maior número de casos ocorreu na região Metropolitana I, com 197.445, sendo 144.199 no município do Rio de Janeiro. O menor número de infectados no período analisado foi no município de Rio das Flores, no Médio Paraíba. 
A figura 1 apresenta o número de casos novos por dia, demostrando um crescente aumento de casos novos registrados a partir de 23 de março.

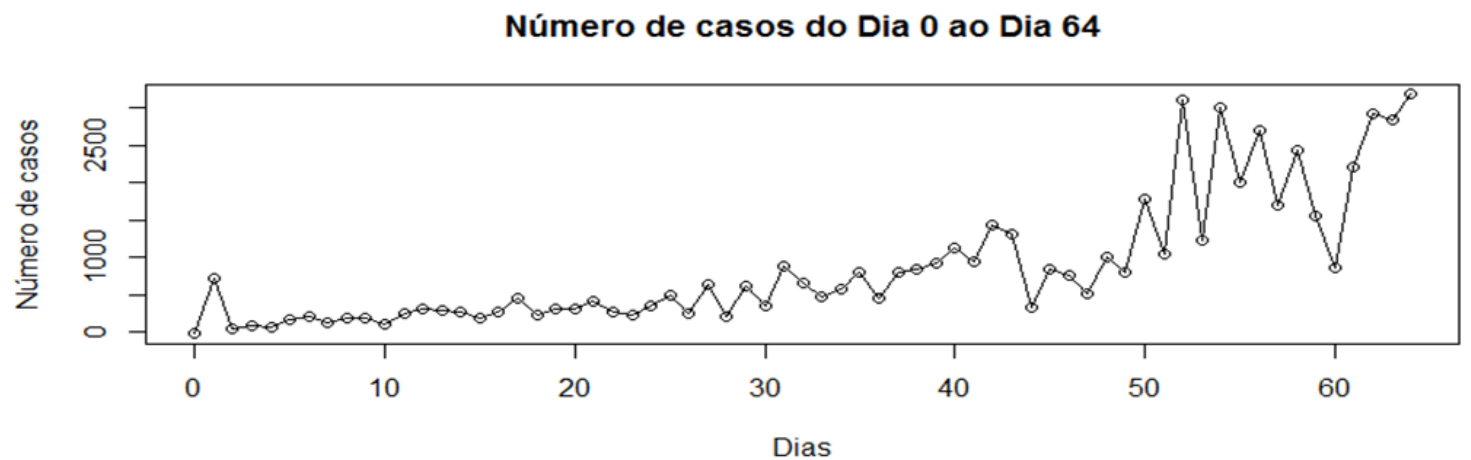

Figura 1. Variação de casos ao longo do período (01/03 a 13/06/2020). Fonte: DataSUS, 2020.

As epidemias seguem um padrão de crescimento exponencial, representando os fenômenos que se multiplicam rapidamente ao longo do tempo. Uma única pessoa infectada é capaz de transmitir um vírus para o seu círculo profissional, familiar e de amizades. Consequentemente, os infectados continuarão a transmissão para seus respectivos grupos de convivência (Lima et al., 2020).

A progressão da doença é acompanhada por meio de um gráfico denominado "curva de contágio", o qual é indicador imprescindível para o investimento e para a organização de fluxos de atendimento da população, criação de hospitais de campanha e investimento em equipamentos de proteção individual para os profissionais de saúde, para evitar redução de profissionais de saúde que podem contrair a doença.

\section{Boxplot Incidência de casos total}

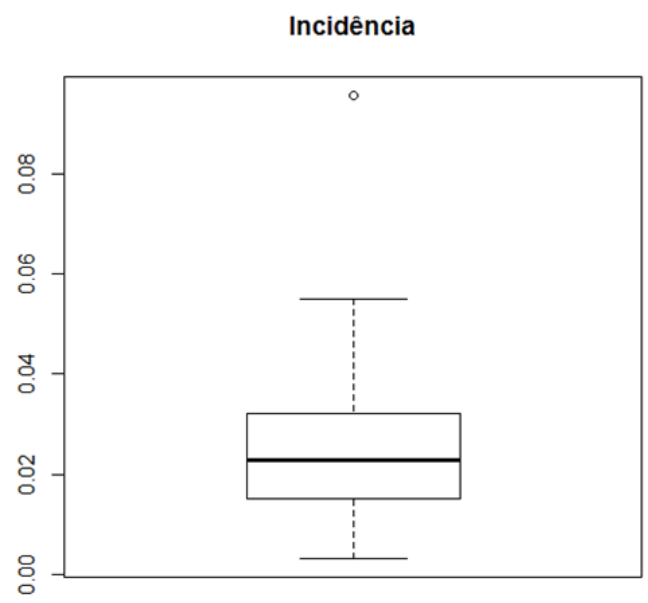

Figura 2. Incidência de casos no Estado do Rio de Janeiro. Fonte: DataSUS, SES-RJ e MS, 2020.

Boxplot de incidência de casos (Figura 2) apresenta um valor atípico muito diferente dos demais. Este valor é representado pelo círculo, em que o município em questão é o de Varre-Sai, com a incidência da doença elevada.

\section{Boxplot - Incidência por Região de saúde}

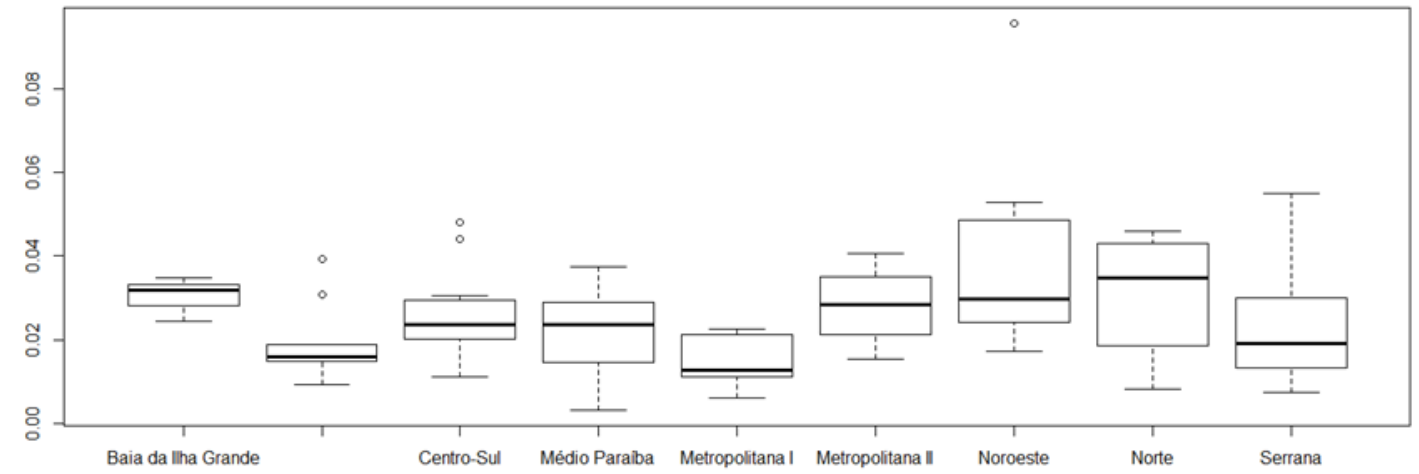

Figura 3. Incidência de casos por Região de Saúde. Fonte: DataSUS, SES-RJ e MS, 2020. 
Observando os boxplots (Figura 3), observa-se o formato da distribuição das incidências pelas diferentes regiões. As medianas variam entre as regiões. O ponto com a maior incidência, no boxplot nomeado "Noroeste" é o município de Varre-Sai.
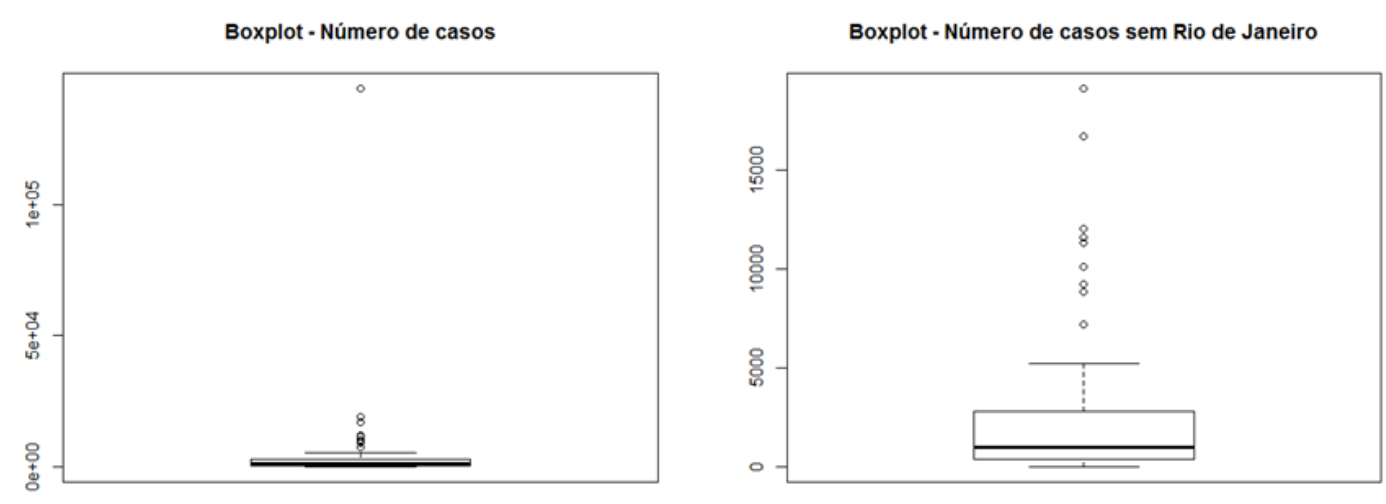

Figura 4. Incidência de casos por Região Metropolitana I. Fonte: DataSUS, SES-RJ e MS, 2020.

Nos Boxplot (Figura 4 e 5) representam a incidência de casos COVID-19, respectivamente, na região Metropolitana 1 e nas regiões de saúde. Na Metropolitana 1 é possível observar que um outlier está mais longínquo que os demais, tal representa o município do Rio de Janeiro. Convém ressaltar, porém que o município concentra 39\% de toda a população do estado do Rio de Janeiro. A distribuição do boxplot é alterada quando o este município é suprimido.

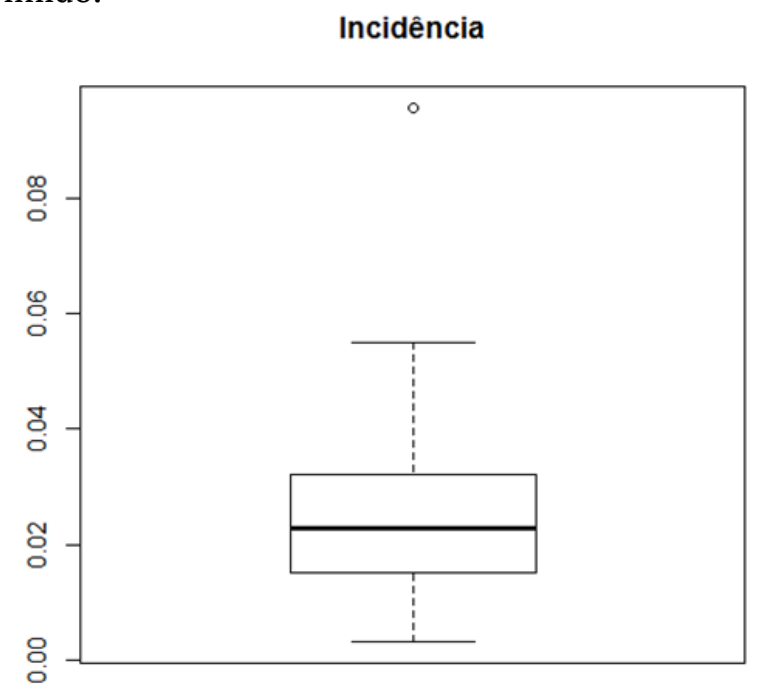

Figura 5. Distribuição de casos por Região de Saúde. Fonte: DataSUS, SES-RJ e MS, 2020.

A figura 6, demonstra que há uma assimetria positiva nas Regiões Baia da Ilha Grande, Médio Paraíba, Centro Sul, Médio Paraíba, Noroeste, Norte e Serrana. As regiões Metropolitana 1 e 2 apresentam uma distribuição mais simétrica.

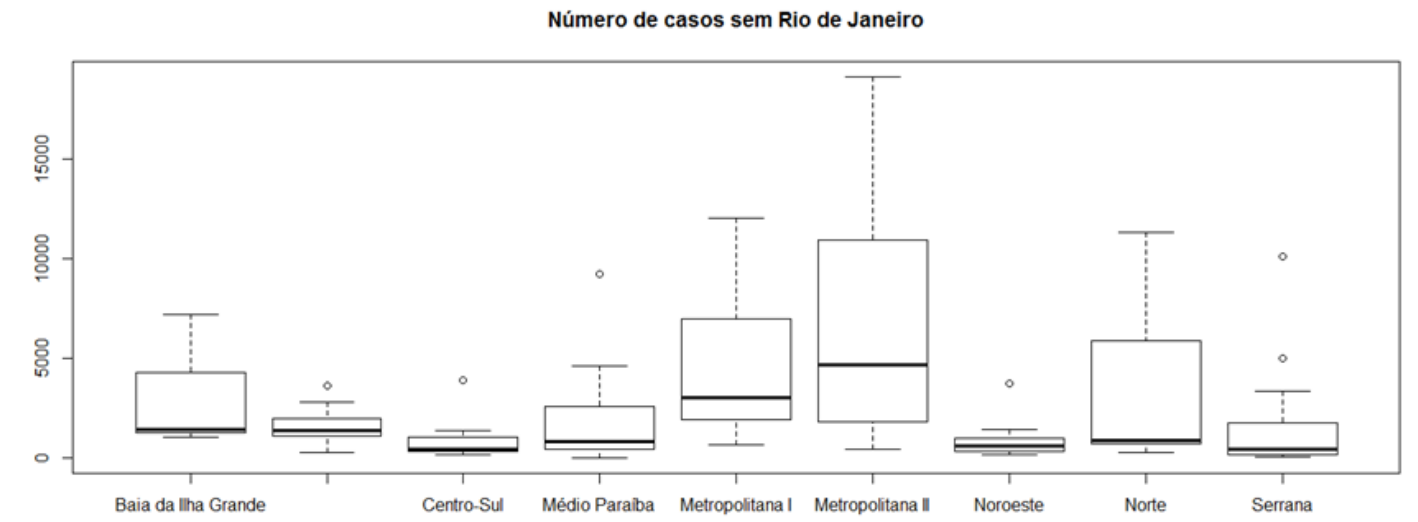

Figura 6. Distribuição de casos excluindo o município do Rio de Janeiro. Fonte: DataSUS, SES-RJ e MS, 2020. 
Há outros valores atípicos em outras regiões: Baixada Litorânea, Centro-Sul, Médio Paraíba, Noroeste e Serrana. As regiões que tiveram as maiores médias foram a Metropolitana I e II, seguido do Norte e da Baia da Ilha Grande. Neste sentido, compreender as redes sociais, econômicas, os padrões comerciais e o fluxo de viagens pode ser importante no entendimento da expansão territorial da doença.

Destaca-se também que as altas médias estão relacionadas a distribuição desigual dos serviços e equipamentos urbanos, a crescente demanda por habitação, acompanhada do aumento de submoradias e da expansão de favelas, a intensa degradação ambiental e o esgotamento dos recursos naturais, a marginalidade, o desemprego e a exclusão social são características deste processo.

Entende-se que para enfrentar uma doença que se propaga muito rapidamente, e não apenas ataca as pessoas, mas compromete o sistema de saúde e a sociedade como um todo, medidas preventivas individuais não são suficientes, e adicionalmente devem ser adotadas medidas de alcance comunitário. Tais medidas incluem restrições ao funcionamento de escolas, universidades, locais de convívio comunitário, transporte público, além de outros locais onde há aglomeração de pessoas, como eventos sociais e esportivos, teatros, cinemas e estabelecimentos comerciais, que não sejam caracterizados como prestadores de serviços essenciais (Remuzzi \& Remuzzi, 2020).

Contudo, há diferentes impactos da doença nas classes sociais, sobretudo nos grupos mais pauperizados. De fato, a mundialização do capital gera ainda mais pobreza, e isso é reconhecido pelos autores de diversas perspectivas teóricas. As diferenças de riqueza entre classes ou indivíduos se reflete em indicadores de saúde, revelando maior gravidade, sobretudo, de certas doenças infecciosas. Esse panorama permite refletir sobre a gravidade com a qual a pandemia pode impactar nas comunidades mais pauperizadas, especialmente nos países de capitalismo dependente, devido ao baixo acesso à água tratada, saneamento e estrutura e renda que permita adotar as medidas de prevenção (Souza, 2020).

A figura 7 apresenta a variação de leitos por região de saúde no estado do Rio de Janeiro.

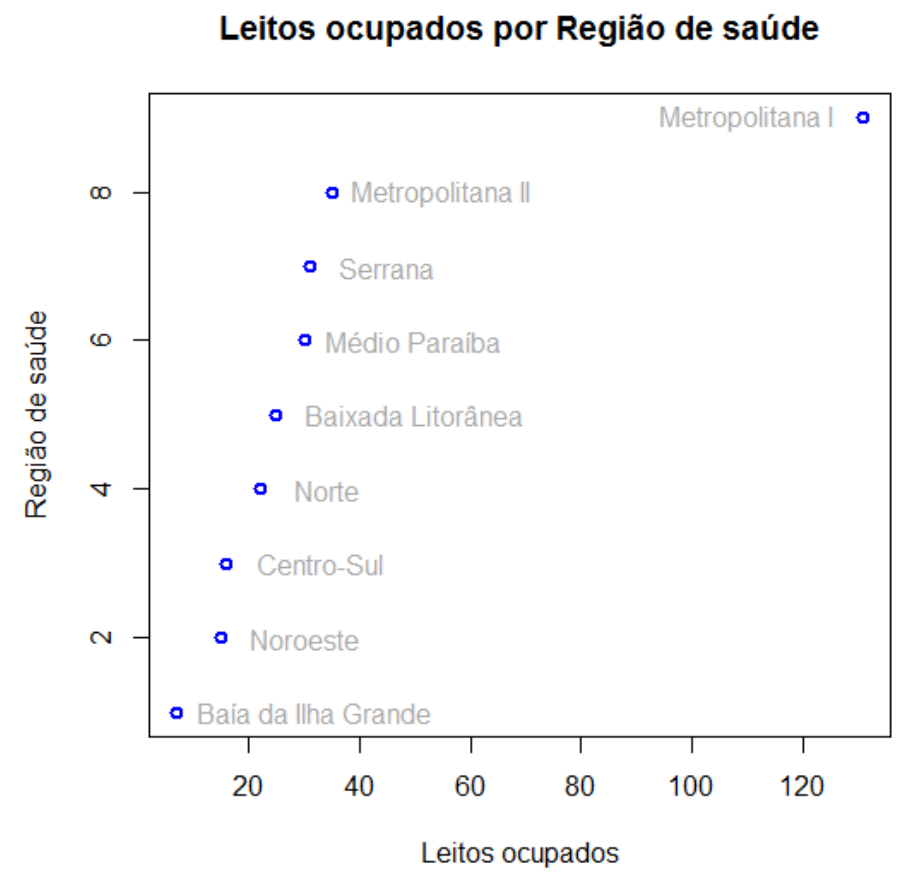

Figura 7. Distribuição de Leitos por Região de Saúde. Fonte: DataSUS, SES-RJ e MS, 2020.

A figura 7 configura a distribuição de leitos ocupados por região de saúde. O município do Rio de Janeiro pertence à região Metropolitana I, é responsável por 30\% dos casos de internação no estado.

Há uma correlação positiva mostrando que o a taxa de óbito está diretamente relacionando ao número de leitos, ou seja, a disponibilidade de leitos está diretamente relacionada ao número de internações, quando há mais disponibilidade de leitos aumenta o número de internações.

A média de leitos por 1.000 habitante no Estado do Rio de Janeiro é de 2,34, ou seja, abaixo do preconizado pela Organização Mundial de Saúde - OMS, segundo levantamentos, datados de 2009, mostram que a oferta corresponde a uma média de 2,4 leitos por mil habitantes, sendo 2,1/mil habitantes no SUS e 2,6 /mil habitantes entre os beneficiários de planos de saúde (AHSEB, 2014). 
A ampliação da estrutura para atendimento dos casos graves requer disponibilidade de leitos de internação e/ou cuidados intensivos, necessitando da aquisição de equipamentos e insumos, da construção de unidades hospitalares, ampliação da capacidade das unidades existentes, contratação de leitos em hospitais privados ou do setor suplementar, bem como do apoio à montagem de hospitais de campanha (Oliveira, 2020).

A inexistência ou fragilidade dos sistemas públicos de saúde não oferece a resiliência necessária para o enfrentamento da pandemia. Apesar do Sistema Único de Saúde (SUS) ser um sistema universal de saúde, com acesso previsto a todos os que necessitam, os governos precisam incluir nos decretos medidas para garantir atendimento público para todos que necessitarem em um contexto que exige o amplo acesso das populações aos serviços de saúde (FIOCRUZ, 2020).

A figura 8 apresenta a relacão entre óbitos e leitos ocupados considerando as regiões de saúde.

\section{Óbitos x Leitos ocupados}

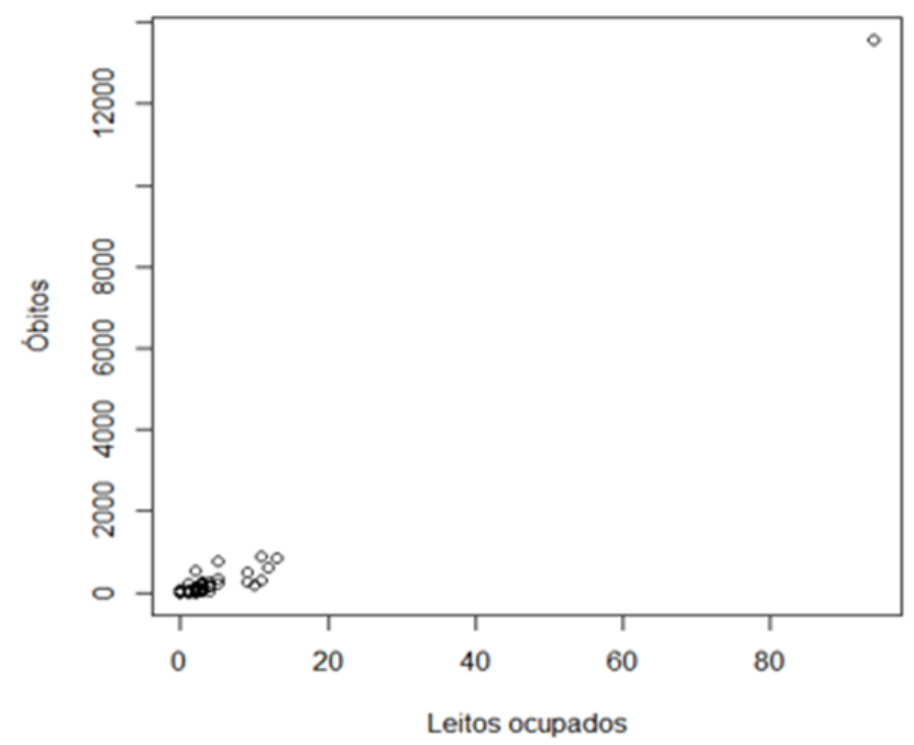

Figura 8. Relação entre óbitos e o número de leitos ocupados. Fonte: DataSUS, SES-RJ e MS, 2020.

A figura 8 demonstra haver uma relação linear positiva entre as variáveis. Calculando a correlação de Pearson, o resultado foi de 0,98 . Ao nível de $5 \%$, confirma-se que o valor da correlação é significativo.

Óbitos x Leitos ocupados (sem município Rio de Janeiro

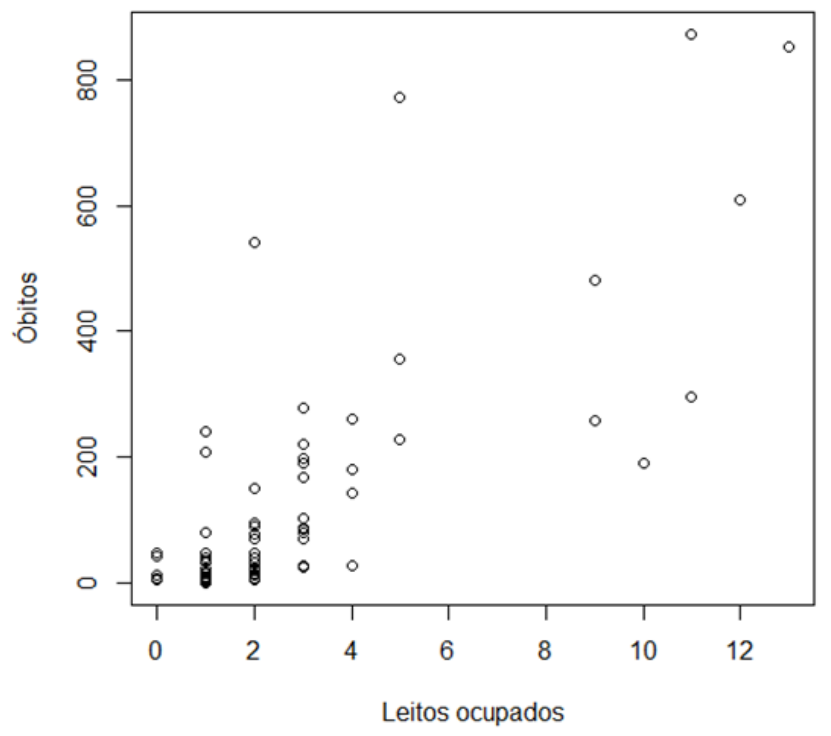

Figura 9. Relação entre óbitos x leitos ocupados excluído município do RJ. Fonte: DataSUS, SES-RJ e MS, 2020.

A figura 9 verifica uma correlação positiva média demonstrando que as variáveis estão envolvidas. Para confirmar a hipótese a correlação foi calculada e o valor apresentado foi de aproximadamente 79\%, o p-valor 
calculado foi baixo, ao nível de significância de 5\%. Assim, embora o valor tenha reduzido, pode-se admitir a existência de relação entre a quantidade de óbitos e a de leitos ocupados.

Outro fator importante a ser destacada é que nas regiões com maiores incidências apresentam uma estrutura de teste para diagnósticos, o que faz com que a doença possa ser detectada ainda no início, o que implica na redução do número de óbitos.

\section{Considerações finais}

Este estudo preocupou-se em apresentar uma visão geral do panorama atual do COVID-19 no estado do Rio de Janeiro e o impacto na prestação da assistência à saúde. Como resultado, fica evidenciado o papel importante da gestão pública, por meio de políticas educativas que busquem conscientizar a população sobre a forma de disseminação do vírus e ampliar as estratégias para identificação rápida da doença, isolamento e tratamento precoce, em virtude de não haver disponibilidade de leitos de internação para atender todos os casos de COVID que evoluam com complicações.

Cumpre destacar que os números de internações hospitalares são influenciados por outros fatores além da morbidade, tais como, disponibilidade de leitos, políticas de admissão hospitalar e fatores sociais. Sendo assim, o debater acerca dos investimentos em gestão de saúde precisa seguir como pauta central, pois é necessário se formar um novo sistema de saúde pós-Covid-19, o que atualmente está sendo um grande desafio, considerando a estrutura dos hospitais e leitos disponíveis no Sistema Único de Saúde.

\section{Referências}

Associação de Hospitais e Serviços de Saúde do Estado da Bahia (AHSEB). 2014. Segundo OMS, ideal é ter de 3 a 5 leitos para cada mil habitantes. No Brasil, índice médio é de 2,4. [S. I.]. Disponível em: http://www.ahseb.com.br/segundo-oms-ideal-e-ter-de-3-a-5-leitos-para-cada-mil-habitantes-nobrasil-indice-medio-e-de-24/. Acesso em: 10 set 2020.

Brasil. Ministério da Saúde. 2020a. Painel de casos de doença pelo coronavírus (COVID-19) no Brasil pelo Ministério da Saúde [Internet]. Brasília: Ministério da Saúde.

Brasil. Ministério da Saúde. Secretaria de Vigilância em Saúde. 2020b. Infecção humana pelo novo coronavírus (2019-nCoV). Brasília: Boletim Epidemiológico, 1-23.

Brasil. Secretaria do Estado do Rio de Janeiro. 2020c. Primeiro caso do Novo Coronavírus é confirmado no Estado do Rio. Disponível em: https://www.saude.rj.gov.br/noticias/2020/03/primeiro-caso-do-novocoronavirus-e-confirmado-no-estado-do-rio. Acesso em: 20 Setembro 2020. Acesso em: 20 set 2020.

Brasil. Ministério da Saúde. 2020d. Resolução ${ }^{\circ}{ }^{1}$, de 29 de setembro de 2011. Estabelece diretrizes gerais para a instituição de Regiões de Saúde no âmbito do Sistema Único de Saúde (SUS), nos termos do Decreto No 7.508, de 28 de junho de 2011. Brasília: Diário Oficial da União.

Brasil. Secretaria de Saúde, Governo do Rio de Janeiro. 2009. Atualização do Plano Diretor de Regionalização 2012-2013, 248p.

Cavalcante, J. R., \& Abreu, A. J. L. 2020. COVID-19 no município do Rio de Janeiro: análise espacial da ocorrência dos primeiros casos e óbitos confirmados. Epidemiologia e Serviços de Saúde, 29(3), e2020204.

Emanuel, E. J., Persad, G., Upshur, R., Thome, B., Parker, M., Glickman, A., Zhang, C., Boyle, C., Smith, M., \& Phillips, J. P. 2020. Fair Allocation of Scarce Medical Resources in the Time of Covid-19. The New England Journal of Medicine, 382, 2049-2055.

Fundação Oswaldo Cruz (FIOCRUZ). 2020. Escola de Saúde Pública. A gestão de riscos e governança na pandemia por Covid-19 no Brasil: análise dos decretos estaduais no primeiro mês. Centro de Estudos e Pesquisas em Emergências e Desastres em Saúde. Disponível em: https://portal.fiocruz.br/sites/portal.fiocruz.br/files/documentos/relatoriocepedes-isolamento-socialoutras-medidas.pdf. Acesso em: 10 set 2020.

Iser, B. P. M., Silva, I., Raymundo, v. T., Poleto, M. B., Shuelter-Trevisol, F., \& Bobinski, F. 2020. Definição de caso suspeito da COVID-19: uma revisão narrativa dos sinais e sintomas mais frequentes entre os casos confirmados. Epidemiologia e Serviços de Saúde, 29(3), e2019354.

Lima, L. D., Pereira, A. M. M., \& Machado, C. V. 2020. Crise, condicionantes e desafios de coordenação do Estado federativo brasileiro no contexto da COVID-19. Cadernos de Saúde Pública, 36(7), e00185220. 
Mattar, F. N. 2001. Pesquisa de marketing ( $3^{\text {a }}$ ed.). São Paulo, SP: Atlas.

Oliveira, W. K. 2020. Como o Brasil pode deter a COVID-19. Epidemiologia e Serviços de Saúde, $29(2)$, e2020044.

Rafael, R. M. R., Neto, M., Carvalho, M. M. B., David, H. M. S. L., Acioli, S., \& Faria, M. G. A. 2020. Epidemiologia, políticas públicas e pandemia de Covid-19: o que esperar no Brasil? Revista Enfermagem UERJ, 28, e49570.

Remuzzi, A., \& Remuzzi, G. 2020. COVID-19 and Italy: what next? The Lancet, 395(10231), 1225-1228.

Richardson, R. J. 1999. Pesquisa social: métodos e técnicas. $3^{a}$ ed. São Paulo: Atlas, 54p.

Souza, D. O. 2020. A pandemia de COVID-19 para além das Ciências da Saúde: reflexões sobre sua determinação social. Ciência \& Saúde Coletiva, 25, 2469-2477.

\section{Minicurrículo}

Tatiana Souza da Silva Werle. Mestranda do Programa de Pós-Graduação em Saúde e Tecnologia no Espaço Hospitalar da Universidade Federal do Estado do Rio de Janeiro - UNIRIO, Pós Graduação em Auditoria em Saúde pela Universidade Estácio de Sá, Residência em Clínica Médica e Cirúrgica pela Universidade Federal do Estado do Rio de Janeiro, Oficial Enfermeira da Marinha do Brasil atuando como Encarregada da Divisão Operacional do Departamento de Auditoria de Contas Médicas, Enfermeira Intensivista e Professora de cursos preparatórios para concursos militares.

Antonio Rodrigues de Andrade. Professor do Departamento de Ciências Sociais e do Programa de Pósgraduação em Saúde e Tecnologia em Espaços Hospitalares da Universidade Federal do Estado do Rio de Janeiro - UNIRIO, Pesquisador Colaborador do Departamento de Gestão da Universidade de Évora com PósDoutorado em Administração pela Universidade do Porto (FEP) e em Ciência da Informação pela UFRJ (IBICT). Doutorado em Engenharia de Transportes (COPPE/UFRJ), Mestrado em Ciência da Informação (IBICT/UFRJ) e Bacharelado em Ciências da Administração (UGF), Especialização em Psicopedagogia (UCAM), MBA em Management (FGV), Especialização em Sistemas de Informação (UNESA) e, complementarmente, Formação na Teoria da Modificabilidade Cognitiva Estrutural (ICELP - International Institute for the Enhancement of Learning Potential - Jerusalém ). Foi professor da PUC, do IBMEC, da UNESA, da ESPM e da FGV já tendo realizado treinamento e consultoria em várias Organizações no Brasil e no Exterior. Conselheiro e Vicepresidente de Educação, Estudos e Pesquisa do CRA-RJ - Conselho Regional de Administração do Rio de Janeiro e Coordenador da Comissão de Empreendedorismo e Inovação e Membro da Câmara Setorial de Formação Profissional e Educação Tecnológica do Fórum de Desenvolvimento Estratégico do Estado do Rio de Janeiro. Possui projetos ligados à comunidade e voluntário da Casa Ronald McDonald. Possui duas linhas de pesquisa. Autor dos livros Ouvindo o Cliente para Mudar, Estratégia, execução e diferenciação baseadas na informação: um estudo em Organizações Brasileira e Portuguesas e Alinhamento Estratégico: textos selecionados (em desenvolvimento).

Como citar: Werle, T.S.S, \& Andrade, A.R. 2021. A pandemia de COVID-19 e os impactos na saúde pública do estado do Rio de Janeiro. Pubsaúde, 5, a128. DOI: https://dx.doi.org/10.31533/pubsaude5.a128

Recebido: 20 jan. 2021.

Revisado e aceito: 29 jan. 2021.

Conflito de interesse: os autores declaram, em relação aos produtos e companhias descritos nesse artigo, não ter interesses associativos, comerciais, de propriedade ou financeiros que representem conflito de interesse.

Licenciamento: Este artigo é publicado na modalidade Acesso Aberto sob a licença Creative Commons Atribuição 4.0 (CC-BY 4.0). 